\title{
ADDENDUM
}

\section{A taxonomic and taphonomic study of Pleistocene fossil deposits from the western Nefud Desert, Saudi Arabia - Addendum}

Mathew Stewart, Julien Louys, Paul S. Breeze, Richard Clark-Wilson, Nick A. Drake, Eleanor M.L. Scerri, Iyad S. Zalmout, Yahya S. A. Al-Mufarreh, Saleh A. Soubhi, Mohammad A. Haptari, Abdullah M. Alsharekh, Huw S. Groucutt, Michael D. Petraglia

DOI: https://doi.org/10.1017/qua.2020.6; Published online by Cambridge University Press 20 March 2020

Additional information for Stewart et al. (2020) inadvertently omitted from the original publication is as follows:

Abdullah M. Alsharekh thanks the Researchers Supporting Project of King Saud University (no. RST-2019/126).

\section{Reference}

Stewart, M., Louys, J., Breeze, P., Clark-Wilson, R., Drake, N., Scerri, E.M.L., Zalmout, I.S., et al. 2020. A taxonomic and taphonomic study of Pleistocene fossil deposits from the western Nefud Desert, Saudi Arabia. Quaternary Research, 1-22. doi:10.1017/qua.2020.6 